\title{
Optimal Homotopy Asymptotic Solution for Exothermic Reactions Model with Constant Heat Source in a Porous Medium
}

\author{
Fazle Mabood ${ }^{1}$ and Nopparat Pochai ${ }^{2,3}$ \\ ${ }^{1}$ Department of Mathematics, University of Peshawar, Peshawar, Pakistan \\ ${ }^{2}$ Department of Mathematics, King Mongkut's Institute of Technology Ladkrabang, Bangkok 10520, Thailand \\ ${ }^{3}$ Centre of Excellence in Mathematics, CHE, Si Ayutthaya Road, Bangkok 10400, Thailand
}

Correspondence should be addressed to Nopparat Pochai; nop_math@yahoo.com

Received 27 May 2015; Accepted 7 June 2015

Academic Editor: John D. Clayton

Copyright (C) 2015 F. Mabood and N. Pochai. This is an open access article distributed under the Creative Commons Attribution License, which permits unrestricted use, distribution, and reproduction in any medium, provided the original work is properly cited.

\begin{abstract}
The heat flow patterns profiles are required for heat transfer simulation in each type of the thermal insulation. The exothermic reaction models in porous medium can prescribe the problems in the form of nonlinear ordinary differential equations. In this research, the driving force model due to the temperature gradients is considered. A governing equation of the model is restricted into an energy balance equation that provides the temperature profile in conduction state with constant heat source on the steady state. The proposed optimal homotopy asymptotic method (OHAM) is used to compute the solutions of the exothermic reactions equation.
\end{abstract}

\section{Introduction}

In physical systems, energy is obtained from chemical bonds. If bonds are broken, energy is needed. If bonds are formed, energy is released. Each type of bond has specific bond energy. It can be predicted whether a chemical reaction would release or need heat by using bond energies. If there is more energy used to form the bonds than to break the bonds, heat is given off. This is well known as an exothermic reaction. On the other hand, if a reaction needs an input of energy, it is said to be an endothermic reaction. The ability to break bonds is activated energy.

Convection has obtained growth uses in many areas such as solar energy conversion, underground coal gasification, geothermal energy extraction, ground water contaminant transport, and oil reservoir simulation. The exothermic reaction model is focused on the system in which the driving force was due to the applied temperature gradients at the boundary of the system. In [1-4], they proposed the investigation of Rayleigh-Bernard-type convection. They also study the convective instabilities that arise due to exothermic reactions model in a porous medium. The exothermic reactions release the heat, create density differences within the fluid, and induce natural convection that turn out the rate of reaction affects [5]. The nonuniform flow of convective motion that is generated by heat sources is investigated by [6-8]. In [9-13], they propose the two- and three-dimensional models of natural convection among different types of porous medium.

In this research, the optimal homotopy asymptotic method for conduction solutions is proposed. The model equation is a steady-state energy balance equation of the temperature profile in conduction state with constant heat source.

The optimal homotopy asymptotic method is an approximate analytical tool that is simple and straightforward and does not require the existence of any small or large parameter as does traditional perturbation method. As observed by Herişanu and Marinca [14], the most significant feature OHAM is the optimal control of the convergence of solutions via a particular convergence-control function $H$ and this ensures a very fast convergence when its components (known as convergence-control parameters) are optimally 
determined. In the recent paper of Herişanu et al. [15] where the authors focused on nonlinear dynamical model of a permanent magnet synchronous generator, in their study a different way of construction of homotopy is developed to ensure the fast convergence of the OHAM solutions to the exact one. Optimal Homotopy Asymptotic Method (OHAM) has been successfully been applied to linear and nonlinear problems $[16,17]$. This paper is organized as follows. First, in Section 2, exothermic reaction model is presented. In Section 3, we described the basic principles of the optimal homotopy asymptotic method. The optimal homotopy asymptotic method solution of the problem is given in Section 4. Section 5 is devoted for the concluding remarks.

\section{Exothermic Reactions Model}

In this section, we introduce a pseudohomogeneous model to express convective driven by an exothermic reaction. The case of a porous medium wall thickness $\left(0<z^{\prime}<L\right)$ is focused. The normal assumption in the continuity and momentum equations in the steady-state energy balance presents a nondimensional form of a BVP for the temperature profile $[5,13]$ :

$$
\frac{d^{2} \theta_{0}}{d z^{2}}+B \phi^{2}\left(1-\frac{\theta_{0}}{B}\right) \exp \left(\frac{\gamma \theta_{0}}{\gamma+\theta_{0}}\right)=0 .
$$

Here, $\theta_{0}$ is the temperature, the parameter $B$ is the maximum feasible temperature in the absence of natural convection, $\phi^{2}$ is the ratio of the characteristic time for diffusion of heat generator, and $\gamma$ is the dimensionless activation energy. In the case of the constant heat source, (1) can be written as

$$
\frac{d^{2} \theta_{0}}{d z^{2}}+B \phi^{2}\left(1-\frac{\theta_{0}}{B}\right)=0,
$$

subject to boundary condition

$$
\begin{gathered}
\frac{d \theta_{0}}{d z}=0, \quad \text { at } z=0, \\
\theta_{0}=0, \quad \text { at } z=1 .
\end{gathered}
$$

\section{Basic Principles of Optimal Homotopy Asymptotic Method}

We review the basic principles of the optimal homotopy asymptotic method as follows.

(i) Consider the following differential equation:

$$
A[u(x)]+a(x)=0, \quad x \in \Omega,
$$

where $\Omega$ is problem domain, $A(u)=L(u)+N(u)$, where $L$, $N$ are linear and nonlinear operators, $u(x)$ is an unknown function, and $a(x)$ is a known function.

(ii) Construct an optimal homotopy equation as

$$
\begin{aligned}
& (1-p)[L(\phi(x ; p))+a(x)] \\
& \quad-H(p)[A(\phi(x ; p))+a(x)]=0,
\end{aligned}
$$

where $0 \leq p \leq 1$ is an embedding parameter and $H(p)=\sum_{i=1}^{m} p^{i} K_{i}$ is auxiliary function on which the convergence of the solution greatly dependent. Here, $K_{j}$ are the convergence-control parameters. The auxiliary function $H(p)$ also adjusts the convergence domain and controls the convergence region.

(iii) Expand $\phi\left(x ; p, K_{j}\right)$ in Taylor's series about $p$, one has an approximate solution:

$$
\begin{array}{r}
\phi\left(x ; p, K_{j}\right)=u_{0}(x)+\sum_{k=1}^{\infty} u_{k}\left(x, K_{j}\right) p^{k}, \\
j=1,2,3, \ldots
\end{array}
$$

Many researchers have observed that the convergence of the series equation (6) depends upon $K_{j},(j=1,2, \ldots, m)$; if it is convergent then, we obtain

$$
\widetilde{v}=v_{0}(x)+\sum_{k=1}^{m} v_{k}\left(x ; K_{j}\right) .
$$

(iv) Substituting (7) in (4), we have the following residual:

$$
R\left(x ; K_{j}\right)=L\left(\widetilde{u}\left(x ; K_{j}\right)\right)+a(x)+N\left(\widetilde{u}\left(x ; K_{j}\right)\right) .
$$

If $R\left(x ; K_{j}\right)=0$, then $\widetilde{v}$ will be the exact solution. For nonlinear problems, generally, this will not be the case. For determining $K_{j},(j=1,2, \ldots, m)$, collocation method, Ritz method, or the method of least squares can be used.

(v) Finally, substituting the optimal values of the convergence-control parameters $K_{j}$ in (7) one can get the approximate solution.

\section{Application of OHAM to an Exothermic Reaction Model}

Applying OHAM on (2), the zeroth, first, and second order problems are

$$
(1-p)\left(\theta_{0}^{\prime \prime}\right)-H(p)\left(\theta^{\prime \prime}+B \phi^{2}\left(1-\frac{\theta_{0}}{B}\right)\right)=0 .
$$

We consider $\theta_{0}, H(p)$ in the following manner:

$$
\begin{aligned}
\theta & =\theta_{0,0}+p \theta_{0,1}+p^{2} \theta_{0,2} . \\
H_{1}(p) & =p K_{1}+p^{2} K_{2},
\end{aligned}
$$

\subsection{Zeroth Order Problem}

$$
\theta_{0,0}^{\prime \prime}=0,
$$

with boundary conditions

$$
\begin{aligned}
& \theta_{0,0}(1)=0, \\
& \theta_{0,0}^{\prime}(0)=0 .
\end{aligned}
$$

The solution of (11) with boundary condition (12) is

$$
\theta_{0,0}(z)=0 \text {. }
$$




\subsection{First Order Problem}

$$
\theta_{0,1}^{\prime \prime}-K_{1} \phi^{2} B=0,
$$

with boundary conditions

$$
\begin{aligned}
& \theta_{0,1}(1)=0 \\
& \theta_{0,1}^{\prime}(0)=0 .
\end{aligned}
$$

The solution of (14) with boundary condition (15) is

$$
\theta_{0,1}\left(z, K_{1}\right)=\frac{K_{1} \phi^{2} B}{2}\left(z^{2}-1\right) \text {. }
$$

\subsection{Second Order Problem}

$$
\begin{aligned}
\theta_{0,2}^{\prime \prime}\left(z, K_{1}, K_{2}\right)= & K_{1} \phi^{2} B+K_{1}^{2} \phi^{2} B-\frac{1}{2} K_{1}^{2} \phi^{4} B z^{2} \\
& +\frac{1}{2} K_{1}^{2} \phi^{4} B+\frac{1}{2} K_{2} \phi^{2} B
\end{aligned}
$$

with boundary conditions

$$
\begin{aligned}
& \theta_{0,2}(1)=0, \\
& \theta_{0,2}^{\prime}(0)=0 .
\end{aligned}
$$

The solution of (17) with boundary condition (18) is

$$
\begin{aligned}
\theta_{0,2}\left(z, K_{1}, K_{2}\right)= & \frac{-1}{24} \phi^{4} K_{1}^{2} B z^{4}+\frac{1}{2} \phi^{2} K_{1} B z^{2} \\
& +\frac{1}{2} \phi^{2} K_{1}^{2} B z^{2}+\frac{1}{4} \phi^{4} K_{1}^{2} B z^{2} \\
& +\frac{1}{2} \phi^{2} K_{2} B z^{2}-\frac{5}{24} \phi^{4} K_{1}^{2} B \\
& -\frac{1}{2} \phi^{2} K_{1} B-\frac{1}{2} \phi^{2} K_{1}^{2} B-\frac{1}{2} \phi^{2} K_{2} B .
\end{aligned}
$$

The final three terms solution via OHAM for $p=1$ is

$$
\begin{aligned}
\widetilde{\theta}_{0}\left(z, K_{1}, K_{2}\right)= & \theta_{0,0}(z)+\theta_{0,1}\left(z, K_{1}\right) \\
& +\theta_{0,2}\left(z, K_{1}, K_{2}\right)
\end{aligned}
$$

The method of least squares is used to determine the convergence control parameters $K_{1}$ and $K_{2}$ in (20). In particular case for $\phi=1, B=10$, the values of the convergence control parameters are $K_{1}=-0.8337205022$ and $K_{2}=$ -0.02092667470 .

By substituting the values of $K_{1}$ and $K_{2}$ in (20) and after simplification, we can obtain the second order approximate solution via OHAM. To check the accuracy of the OHAM solution, a comparison between the solutions determined by OHAM and numerical methods was made and is presented in Table 1. Graphical representation of the solution using finite difference technique [5], OHAM, and Runge-Kutta Fehlberg fourth fifth order method is shown in Figure 1; an excellent
TABLE 1: Comparison of $\theta_{0}(z)$ via OHAM and RKF45 for $\phi=1, B=$ 10 .

\begin{tabular}{lcccc}
\hline$Z$ & FDM [5] & RKF45 & OHAM & Percentage error \\
\hline 0.0 & 3.114344 & 3.518277 & 3.518285 & 0.000227 \\
0.1 & 3.046176 & 3.485927 & 3.485969 & 0.001204 \\
0.2 & 2.911251 & 3.388613 & 3.388675 & 0.001829 \\
0.3 & 2.711819 & 3.225339 & 3.225359 & 0.000620 \\
0.4 & 2.451166 & 2.994264 & 2.994284 & 0.000667 \\
0.5 & 2.133897 & 2.693071 & 2.693037 & 0.001262 \\
0.6 & 1.766284 & 2.318441 & 2.318432 & 0.000388 \\
0.7 & 1.356680 & 1.866723 & 1.866701 & 0.001178 \\
0.8 & 0.915960 & 1.333395 & 1.333311 & 0.006299 \\
0.9 & 0.457980 & 0.713042 & 0.713046 & 0.000560 \\
1.0 & 0.000000 & 0.000000 & 0.000000 & -
\end{tabular}

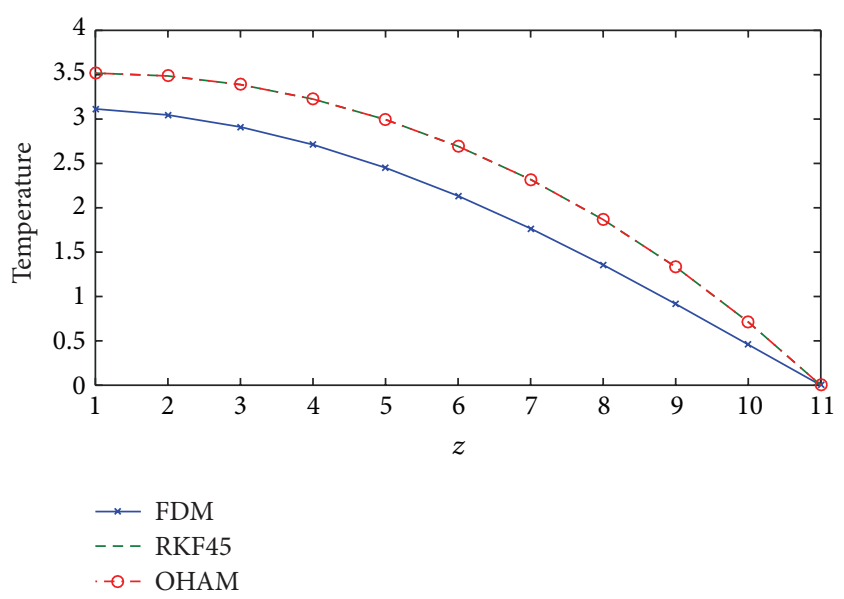

FIGURE 1: Comparison of analytical and numerical solution.

agreement can be observed. We can see that the OHAM gives a better accurate solution than the traditional finite difference technique of [5]. On the other hand, the OHAM gives a continuity solution, but the traditional finite difference technique gives a discrete solution. It follows that the solutions of the OHAM is easier to implement than the finite difference solutions.

In Figure 2, we exhibit the effect of different values of $\phi$ with fixed value of $B$ on temperature profile.

\section{Concluding Remarks}

In this paper, one has described an optimal homotopy asymptotic technique for obtaining the temperature profiles in porous medium. We can see that the temperature reduces to the end. The OHAM scheme for obtaining the model is convenient to implement. The OHAM gives fourth order accurate solutions. It follows that the method has no instability problem. The model should be considered in the case of nonconstant heat source. 


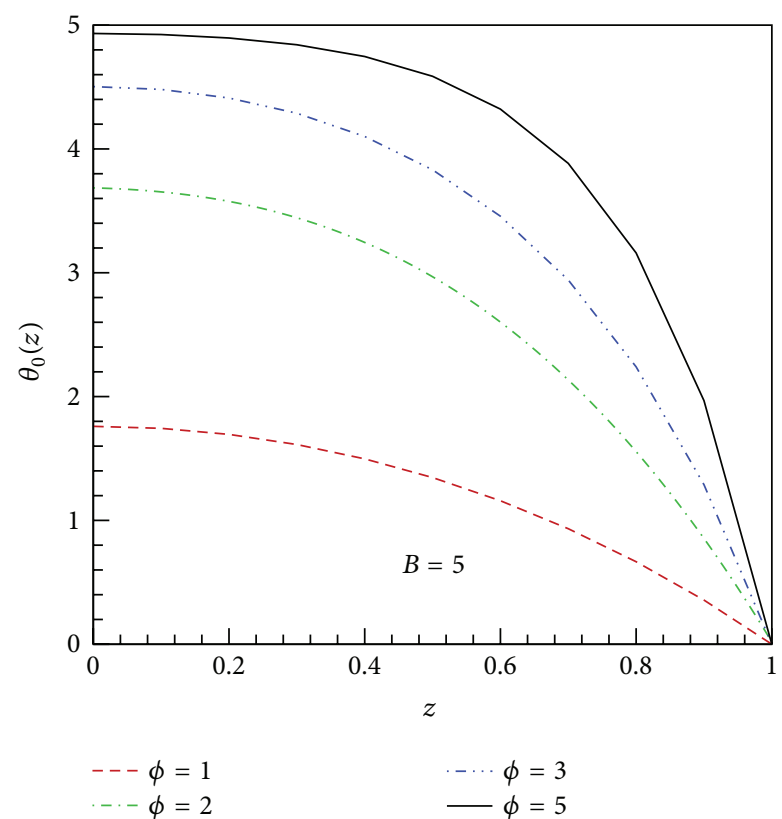

FIgURE 2

\section{Conflict of Interests}

The authors declare that there is no conflict of interests regarding the publication of this paper.

\section{Acknowledgments}

This paper is supported by the Centre of Excellence in Mathematics, the Commission on Higher Education, Thailand. The authors greatly appreciate valuable comments received from Professor John D. Clayton and their reviewers.

\section{References}

[1] J. L. Beck, "Convection in a box of porous material saturated with fluid," Physics of Fluids, vol. 15, no. 8, pp. 1377-1383, 1972.

[2] S. H. Davis, "Convection in a box: linear theory," Journal of Fluid Mechanics, vol. 30, no. 3, pp. 465-478, 1967.

[3] Z. Gershuni and E. M. Zhukovitskii, Convective Stability of Incompressible Fluids, vol. 4, Israel Program for Scientific Translations, 1976.

[4] E. R. Lapwood, "Convection of a fluid in a porous medium," Proceedings of the Cambridge Philosophical Society, vol. 44, pp. 508-521, 1948.

[5] N. Pochai and J. Jaisaardsuetrong, "A numerical treatment of an exothermic reactions model with constant heat source in a porous medium using finite difference method," Advanced Studies in Biology, vol. 4, no. 6, pp. 287-296, 2012.

[6] D. R. Jones, "The dynamic stability of confined, exothermically reacting fluids," International Journal of Heat and Mass Transfer, vol. 16, no. 1, pp. 157-167, 1973.

[7] M. Tveitereid, "Thermal convection in a horizontal porous layer with internal heat sources," International Journal of Heat and Mass Transfer, vol. 20, no. 10, pp. 1045-1050, 1977.
[8] J. B. Bdzil and H. L. Frisch, "Chemically driven convection," The Journal of Chemical Physics, vol. 72, no. 3, pp. 1875-1886, 1980.

[9] H. Viljoen and V. Hlavacek, "Chemically driven convection in a porous medium," AIChE Journal, vol. 33, no. 8, pp. 1344-1350, 1987.

[10] H. J. Viljoen, J. E. Gatica, and H. Vladimir, "Bifurcation analysis of chemically driven convection," Chemical Engineering Science, vol. 45, no. 2, pp. 503-517, 1990.

[11] W. W. Farr, J. F. Gabitto, D. Luss, and V. Balakotaiah, "Reactiondriven convection in a porous medium," AIChE Journal, vol. 37, no. 7, pp. 963-985, 1991.

[12] K. Nandakumar and H. J. Weinitschke, "A bifurcation study of chemically driven convection in a porous medium," Chemical Engineering Science, vol. 47, no. 15-16, pp. 4107-4120, 1992.

[13] S. Subramanian and V. Balakotaiah, "Convective instabilities induced by exothermic reactions occurring in a porous medium," Physics of Fluids, vol. 6, no. 9, pp. 2907-2922, 1994.

[14] N. Herisanu and V. Marinca, "Accurate analytical solutions to oscillators with discontinuities and fractional-power restoring force by means of the optimal homotopy asymptotic method," Computers \& Mathematics with Applications, vol. 60, no. 6, pp. 1607-1615, 2010.

[15] N. Herisanu, V. Marinca, and G. Madescu, "An analytical approach to non-linear dynamical model of a permanent magnet synchronous generator," Wind Energy, 2014.

[16] F. Mabood and N. Pochai, "Asymptotic solution for a water quality model in a uniform stream," International Journal of Engineering Mathematics, vol. 2013, Article ID 135140, 4 pages, 2013.

[17] V. Marinca and N. Herisanu, "Optimal homotopy perturbation method for strongly nonlinear differential equations," Nonlinear Science Letters A, vol. 1, no. 3, pp. 273-280, 2010. 


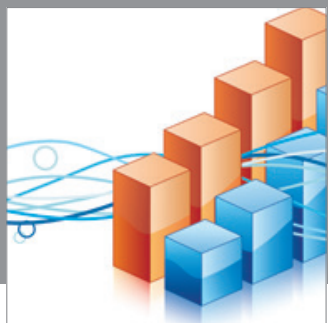

Advances in

Operations Research

mansans

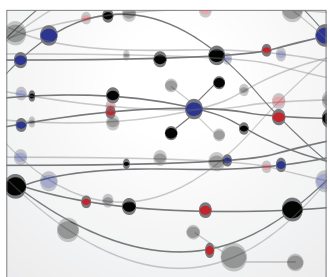

The Scientific World Journal
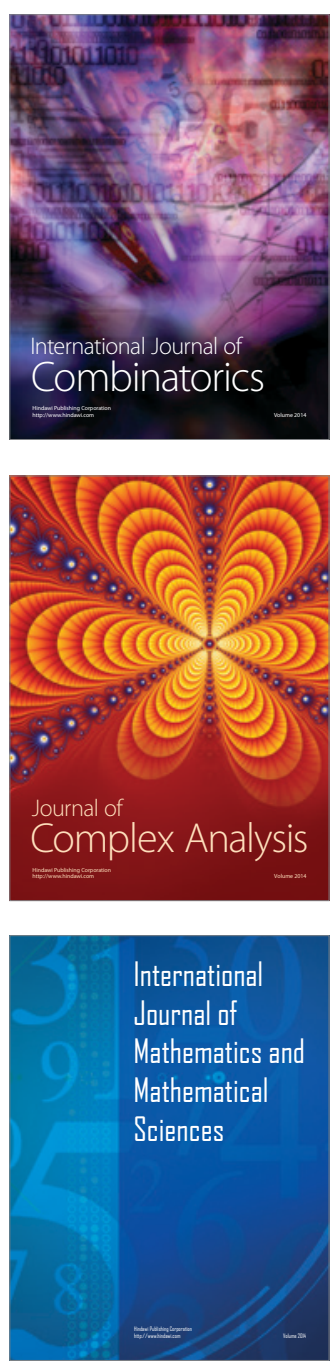
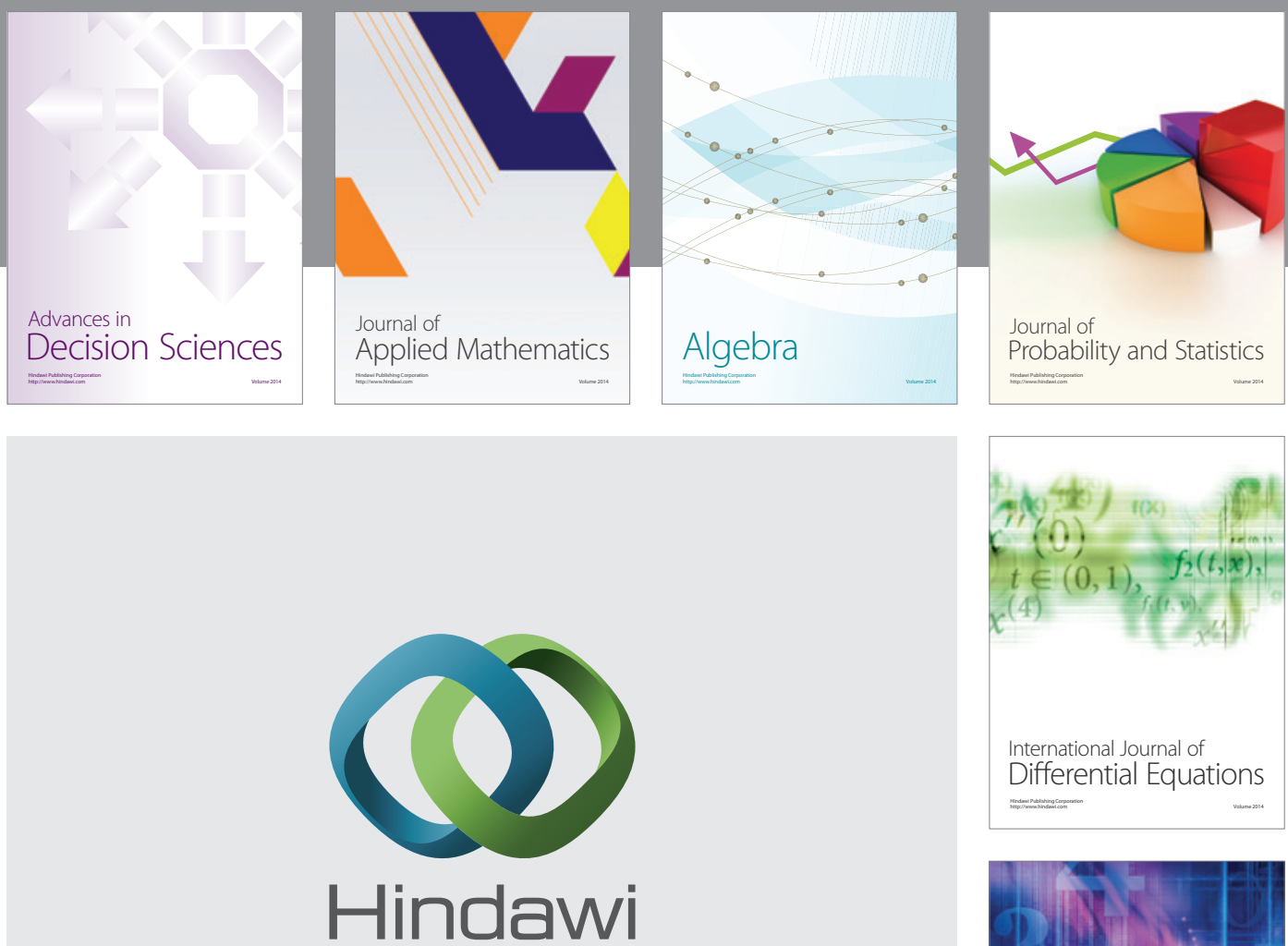

Submit your manuscripts at http://www.hindawi.com
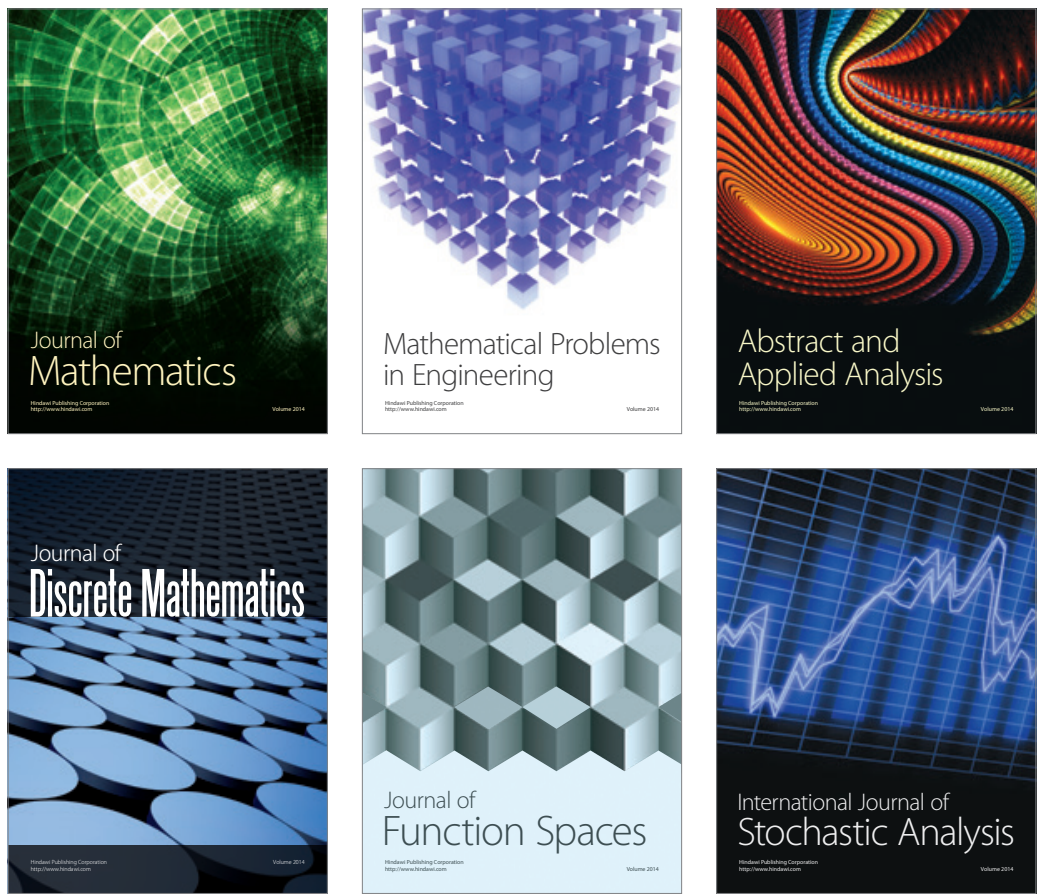

Journal of

Function Spaces

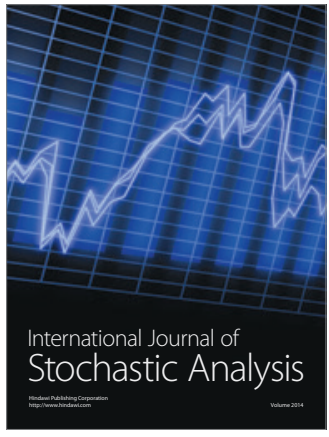

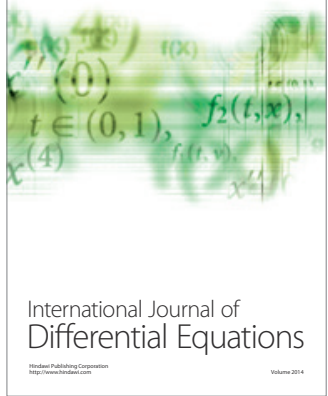
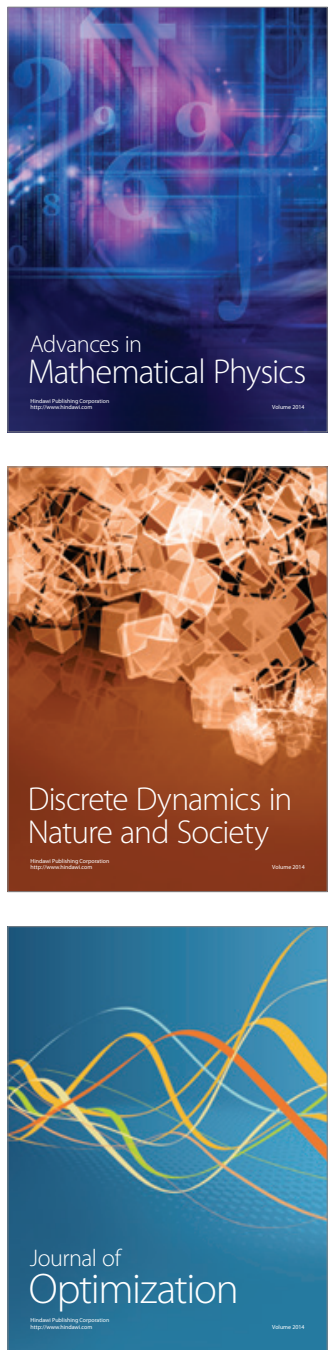